\title{
The Influence of Sound Deprivation on Audiologic Outcomes after Cochlear Implantation
}

\author{
Maria Stella Arantes do Amaral*, Ana Cláudia Mirândola Barbosa Reis, Henrique F. Pauna, \\ Sarah C. Bernal, Eduardo T. Massuda, Miguel Angelo Hyppolito
}

Department of Ophthalmology, Otorhinolaryngology, Head and Neck Surgery, Ribeirão Preto Medical School, University of São Paulo (FMRP-USP), Ribeirão Preto, São Paulo, Brazil

Email:*stella_arantes@yahoo.com, *mariastella_amaral@usp.br

How to cite this paper: do Amaral, M.S.A., Reis, A.C.M.B., Pauna, H.F., Bernal, S.C., Massuda, E.T. and Hyppolito, M.A. (2018) The Influence of Sound Deprivation on Audiologic Outcomes after Cochlear Implantation. International Journal of Clinical Medicine, 9, 28-38. https://doi.org/10.4236/ijcm.2018.91004

Received: November 13, 2017 Accepted: January 14, 2018 Published: January 17, 2018

Copyright (c) 2018 by authors and Scientific Research Publishing Inc. This work is licensed under the Creative Commons Attribution International License (CC BY 4.0).

http://creativecommons.org/licenses/by/4.0/

\begin{abstract}
The cochlear implant is a prosthesis that is capable to replace listening capability among patients with sensorineural hearing loss. Many studies suggest a negative relationship among duration of sound deprivation and audiometric gain (by means of speech perception test) after cochlear implantation. We evaluated surgical and audiologic results among post-lingual deafened adults, in different times of sound deprivation, and correlated with social isolation, presence of vertigo or tinnitus, and the speech therapy yield, between pre- and post-operative stages. A retrospective study was conducted on patients with post-lingual hearing loss, aged over 18 years old who underwent cochlear implant surgery in a public hospital from 2004 to 2014. Audiological, sociodemographic questionnaire, and manifestations of vertigo and tinnitus, during pre- and post-operative stages, were compared and correlating to the different periods of sound deprivation. Two groups were studied. The group $A$ was composed of 38 participants ( 14 male and 24 female) with sound deprivation of less than 10 years; and group $B$ was composed of 17 participants ( 8 male and 9 female) with sound deprivation longer than 10 years. We found no differences regarding age, sex, duration of sound deprivation, mean pure-tone audiometric results (pre- and post-surgery), and speech perception tests (pre-operatively). After one year of the surgery, we observed a significant difference between both groups, with a better performance to group $A$. The results of speech perception test, after cochlear implantation in group $B$, were worse than the results obtained in group $A$, although these correlations are not determinant whether to perform cochlear implant surgery or not.
\end{abstract}




\section{Keywords}

Audiometry, Cochlear Implantation, Sensorineural Hearing Loss, Sound Deprivation, Deafness

\section{Introduction}

Sensorineural hearing loss (SNHL), no matter of its severity, may lead to a series of changes in reception and audiologic comprehension, and consequently, in acquisition of oral language, since deafness hinders audiologic feedback, making it difficult for the individual to modulate his/her own voice. Hearing loss can also lead to impairment of the individual's communication, and can cause secondary issues involving other aspects, such as cognitive, emotional and social [1] [2].

Cochlear implant (CI) is a prosthesis that partially replaces the auditory organ. It is indicated for children and adults with severe to profound SNHL, when the benefit of hearing aid (HA) is no longer achieved. The HA acts as a sound amplifier that requires a sufficient cochlear reserve to allow the amplified sound stimulus to be received. Because it is a sensory prosthesis, the CI can benefit people with different degrees of hearing loss, and with different periods of sound deprivation [3] [4] [5].

A factor to be considered for the indication of the CI surgery, besides the degree and time of acquisition of the hearing loss, is the time of sound deprivation, that is the elapsed time in which the individual remained with no auditory stimulus (with no use of HA or any other device that allowed sound signal input). A negative correlation was observed between the duration of sound deprivation and audiometric gain (by means of speech perception tests post-operatively), although it does not contraindicate CI surgery to be performed [6] [7] [8].

Geier et al. [9] reported that deaf patients for over $60 \%$ of their years of age presented a slower improvement of their speech recognition. In the meantime, Blamey et al. [10] and Plant et al. [11] reported that auditory improvement is inversely proportional to duration of deafness. Yet, Moon et al. [12] reported data showing favorable auditory prognosis among patient that developed deafness after youth even with longer periods of sound deprivation.

Studies have shown that about $90 \%$ of patients present tinnitus and hearing loss. Chronic tinnitus is seen in about $65 \%$ of patients with SNHL, $5 \%$ of patients with mixed hearing loss, and $4 \%$ of conductive hearing loss cases. Most of the evidence shows no relationship between the severity of hearing loss and the annoyance generated by the tinnitus, however, it is known that hearing loss and tinnitus can be functionally detrimental to daily life, since they lead to social isolation and communication disturbances, leading to worsening quality of life [13] [14] [15] [16] [17].

The symptom generator may be present at various levels of the auditory sys- 
tem-at the cochlear or retrocochlear level-and is frequently related to damage to the peripheral auditory system. However, the pathophysiology of tinnitus is still not fully elucidated and the heterogeneity of findings in the literature does not allow a breakthrough in specific treatments for all cases. There is a consensus among researchers about the need for studies with homogenous populations, or diseases with common aspects to decrease the risk of bias, increasing the chances to clarify the pathophysiology of tinnitus, and to provide human hearing comfort [14] [18] [19].

Recent studies and clinical practice show that patients with hearing loss (of any types or degrees) associated to tinnitus are highly benefited with the use of HA, either immediately to HA fitting, or short to medium term. The HA adaptation for tinnitus increases the levels of understanding of the conversation and alleviates the perception of tinnitus, when performed properly [13] [14] [20] [21].

The knowledge of the tests and criteria which may provide data regarding the prognosis of the auditory performance among candidates for the CI has been the greatest challenge of the teams of professionals who work in this area. Yet, the knowledge applied looking for the improvement of tinnitus after cochlear implantation, and its correlation to the time of sound deprivation and time of hearing loss may, therefore, improve the counseling of CI candidates with tinnitus.

The objective of the present study is to evaluate surgical and auditory results in post-lingual deafened adult patients, with severe to profound bilateral SNHL in different times of sound deprivation, to correlate these results to social isolation, speech therapy, and presence of vertigo and tinnitus in the pre- and post-operative stages, and to verify the possibility of expanding the benefits of CI to post-lingual patients with time of sound deprivation greater than 10 years.

\section{Materials and Methods}

This is a longitudinal retrospective study based on the review and analysis of medical records of post-lingual deafened patients submitted to CI surgery at a public hospital from 2004 to 2014, with the approval of the Research Ethics Committee, number 60,964,416.0.0000.5440.

The auditory health program of this public hospital is under an accredited conformity and legally regulation by the Ministry of Health of the country as a reference service, of high complexity, and performs an average of one hundred cochlear implant surgeries per year.

During the pre-operative period, the patient was evaluated by a team of professionals, as instructed by the following regulations: GM 79 of April 24, 2012 (available at

http://bvsms.saude.gov.br/bvs/saudelegis/gm/2012/prt0793_24_04_2012.html, last access on November 20, 2017), and GM 835 of April 25, 2012 (available at http://bvsms.saude.gov.br/bvs/saudelegis/gm/2012/prt0835_25_04_2012.html, 
last access on November 20, 2017).

Data during pre-and post-operative stages were obtained from the patient's records, and information regarding age at cochlear implantation, sex, age of the onset of hearing loss, duration of sound deprivation, results of the audiological evaluation (auditory thresholds and speech perception test), social isolation, labor inclusion, adherence to the speech therapy process, presence of tinnitus and vertigo, and satisfaction regarding the use of $\mathrm{CI}$, were registered by our team. For each aspect related to hearing (auditory thresholds, and speech perception tests) data were recorded by the speech therapist; for the aspects related to social and labor issues, by the psychologist; and so on. It was standardized a period of one and two years after the surgery to collect the aforementioned data.

It has been proven that the quality of health services includes not only the professional training, the available technologies and the intervention process itself. The quality of the documentation and the registration of all the actions of the professionals involved must also be considered. In this service, protocols were developed and every team of professionals has been constantly oriented to the importance of filling it, with written information that reflects the care and treatment provided, in order to establish an effective communication between the Hearing Health Program team and the other professionals involved in the care of the patient.

Despite the considerations mentioned on the importance of the legal and assistance aspects, some annotations could not represent the right information necessary for this study, which made the inclusion of these patients infeasible or limited the interpretation in considering them for the statistical study.

Adult patients (older than 18 years of age), with unilateral CI, and fitted with the device for at least one year were included. Whilst patients with bilateral or binaural CI, pre-lingual hearing loss, younger than 18 years of age, any changes in neurodevelopment, psychomotor, or psychiatric, and patients with history of meningitis or central nervous system involvement were excluded from this study.

After chart review, patients were divided into two groups: group $A$, composed of patients with sound deprivation of less than 10 years, and group $B$, with sound deprivation greater than 10 years.

Data related to quantitative variables were presented by mean and standard deviation (mean $\pm \mathrm{SD}$ ) or median and interquartile range (IIQ: percentile 75 percentile 25), and categorical variables by means of percentage. Comparisons between groups were performed by Student's t test for independent samples, and Mann-Whitney $U$ test was used when non-parametric analysis were required. For categorical variables, qui-square and $M c N e m a r^{2} s$ tests were used, and lastly, to compare different scores, Mann-Whitney $U$ test were used. All statistical analyzes were performed using $\mathrm{JMP}^{\circledR}$ Software 10.0 (SAS Institute Inc., Cary, NC, USA). Findings were considered statistically significant when $P$ values were less than 0.05 . 


\section{Results}

Group $A$ was composed of 38 patients (14 male and 24 female), mean age of 41.9 \pm 15.8 years. Group $B$ was composed of 17 patients ( 8 male and 9 female), mean age of $43.5 \pm 15.8$ years. No difference was observed related to sex $(P=0.4748$, chi-square test) or age ( $P=0.7334$, Student's t test) between groups (Table 1$)$.

The time of hearing loss was compatible with the duration of sound deprivation, and there was a statistically significant difference between groups (group $A$ : $17.7 \pm 12.5$ years vs. group $B: 27.1 \pm 9.1$ years; $P=0.0031$; Figure 1 ).

When auditory thresholds were analyzed (considering mean auditory thresholds at 500,1000, 2000, and $4000 \mathrm{~Hz}, \mathrm{dBNA}$ ) pre-operatively, by supra aural phones, and post-operatively, by free-field audiometry, there was no differences between group $A$ and group $B(P=0.1796$ [pre-operatively], and $P=0.3138$ [post-operatively], Mann-Whitney $U$ test). Yet, there was no difference between both groups when auditory gain after CI was analyzed $(P=0.1087)$. However, when each group was taken separately, auditory gain after surgery was statistically significant $(P<0.0001$, Table 2$)$.

When speech perception tests were performed pre-operatively (expressed by percentage of correct answers), there was no difference between group $A$ (8.5 \pm $15.4)$ and group $B(10.8 \pm 16.9 ; P=0.4946$, Mann-Whitney $U$ test $)$. After one year of the surgery, speech perception tests were significantly higher within group $A(91.2 \pm 9.0)$ than group $B(78.8 \pm 17.1 ; P=0.0163$, Mann-Whitney $U$ test), as demonstrated in Figure 2 and Table 3.

There were no differences between groups A and B related to the etiology of hearing loss (Table 4).

The characteristics of social isolation, adherence to speech therapy, and labor inclusion were not statistically different during pre- and post-operative stages, between groups. It was found a statistical difference when user's satisfaction of

Table 1. Age and sex of participants $(n=55)$.

\begin{tabular}{ccccc}
\hline \multirow{2}{*}{ Group } & N & \multicolumn{2}{c}{ Sex } & $\begin{array}{c}\text { Age at the time of } \\
\text { surgery (years) }\end{array}$ \\
\cline { 3 - 5 } A & 38 & $14(36.8 \%)$ & $24(63.2 \%)$ & $41.9 \pm 15.8$ \\
B & 17 & $8(47.1 \%)$ & $9(52.9 \%)$ & $43.5 \pm 15.8$ \\
$\boldsymbol{P}$ & & & $0.4748^{*}$ & $0.7334^{* *}$ \\
\hline
\end{tabular}

$\mathrm{A}=$ participants with sound deprivation of less than 10 years; $\mathrm{B}=$ participants with sound deprivation greater than 10 years; $\mathrm{N}=$ total number of participants in each group; ${ }^{*}=$ chi-square test ${ }^{* *}=$ Student's $t$ test.

Table 2. Mean auditory thresholds, in dBNA, pre- and post-cochlear implantation.

\begin{tabular}{ccccc}
\hline Group & Pre-CI & Post-CI & Gain & $P(* *)$ \\
\hline A & $114.2 \pm 7.6$ & $29.5 \pm 6.5$ & $84.6 \pm 10.0$ & $<0.0001$ \\
B & $111.5 \pm 8.0$ & $32.4 \pm 8.5$ & $79.1 \pm 12.0$ & $<0.0001$ \\
$P(*)$ & 0.1796 & 0.3138 & 0.1087 & \\
\hline
\end{tabular}

Post-CI $=$ post-operative stage; Pre-CI $=$ pre-operative stage; ${ }^{*}=$ comparison between groups by Mann-Whitney $U$ test ${ }^{* *}=$ comparison pre- and post-operatively, by Wilcoxon rank sum test. 
Table 3. Speech perception tests, pre- and post-cochlear implantation.

\begin{tabular}{cccc}
\hline Group & SPT Pre-CI (\%) & SPT Post-CI (\%) & $P\left({ }^{* *}\right)$ \\
\hline A & $8.5 \pm 15.4$ & $91.2 \pm 9.0$ & $<0.0001$ \\
B & $10.8 \pm 16.9$ & $78.8 \pm 17.1$ & $<0.0001$ \\
$\boldsymbol{P ( * )}$ & 0.4946 & 0.0163 & \\
\hline
\end{tabular}

SPT Post-CI = post-operative speech perception test; SPT Pre-CI = pre-operative speech perception test; ${ }^{*}=$ comparison between groups by Mann-Whitney $U$ test, ${ }^{* *}=$ comparison pre- and post-operatively, by Wilcoxon rank sum test.

Table 4. Etiology of hearing loss $(n=55)$.

\begin{tabular}{cccc}
\hline Etiology & Group A N and (\%) & Group B N and (\%) & Total N and (\%) \\
Genetic & $4(40)$ & $6(60)$ & $10(18.2)$ \\
Idiopathic & $14(87.5)$ & $2(12.5)$ & $16(29.1)$ \\
Infectious & $6(54.6)$ & $5(45.4)$ & $11(20)$ \\
Otosclerosis & $4(80)$ & $1(20)$ & $5(9.1)$ \\
Ototoxicity & $1(50)$ & $1(50)$ & $2(3.6)$ \\
Sudden deafness & $1(100)$ & $0(0)$ & $1(1.8)$ \\
Trauma & $8(80)$ & $2(20)$ & $10(18.2)$ \\
\hline
\end{tabular}

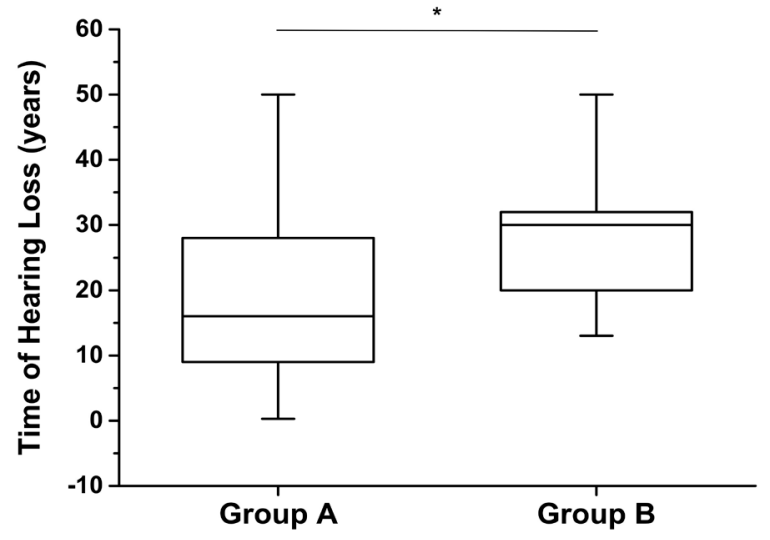

Figure 1. Correlation between time of hearing loss (years) and duration of sound deprivation $(P=0.0031$ - Student's $t$ test, $t=$ $31,355)$ between groups $A$ and $B .{ }^{*}=$ statistically significant.

CI was compared between group $A$ and group $B(P=0.0077$, chi-square test, Table 5).

Tinnitus was identified in 20 out of 38 patients (52.63\%) of group A, pre-operatively. Only one patient of this same group presented tinnitus after surgery $(P<0.0001, M c N e m a r ' s$ test $)$. This corresponds an improvement of $95 \%$ of this complaint. It was also identified in 11 out of 17 patients $(64.7 \%)$ of group $B$, pre-operatively. After surgery, three patients still complained of tinnitus $(P=$ $0.0196, M c N e m a r$ 's test $)$. In the group of 11 patients with tinnitus in pre-operative stage, it was observed that $27.3 \%$ kept them complains after surgery.

Symptoms of vertigo were identified in $12(31.6 \%)$ patients of group $A$ before cochlear implantation, and $6(15.8 \%)$ of them kept the symptoms after surgery 


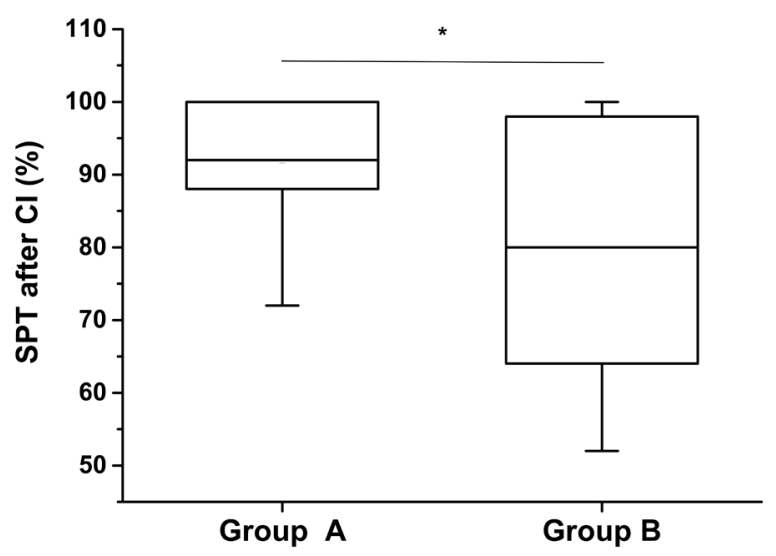

Figure 2. Speech perception tests comparisons between groups $(\mathrm{P}=0.0163$, Mann-Whitney $U$ test $)$. SPT $=$ speech perception test; ${ }^{*}=$ statistically significant.

Table 5. Results of the variables comparing pre- and post-cochlear implantation related to social aspects of the study.

\begin{tabular}{|c|c|c|c|}
\hline \multirow{2}{*}{ Variable } & \multicolumn{2}{|c|}{ Group } & \multirow{2}{*}{$P\left({ }^{*}\right)$} \\
\hline & A & B & \\
\hline \multicolumn{4}{|c|}{ Social isolation (pre-operative) } \\
\hline Yes & $14(40 \%)$ & $6(37.5 \%)$ & \multirow{2}{*}{0.8653} \\
\hline No & $21(60 \%)$ & $10(62.5 \%)$ & \\
\hline \multicolumn{4}{|c|}{ Social isolation (post-operative) } \\
\hline Yes & $3(8.8 \%)$ & $1(6.7 \%)$ & \multirow{2}{*}{0.7959} \\
\hline No & $31(91.2 \%)$ & $14(93.3 \%$ & \\
\hline \multicolumn{4}{|c|}{ Labor inclusion (pre-operative) } \\
\hline Yes & $20(55.6 \%)$ & $10(62.5 \%)$ & \multirow{2}{*}{0.6399} \\
\hline No & $16(44.4 \%)$ & $6(37.5 \%)$ & \\
\hline \multicolumn{4}{|c|}{ Labor inclusion (post-operative) } \\
\hline Yes & $26(74.3 \%)$ & $13(92.9 \%)$ & \multirow{2}{*}{0.1451} \\
\hline No & $9(25.7 \%)$ & $1(7.1 \%)$ & \\
\hline \multicolumn{4}{|c|}{ Speech therapy adherence (pre-operative) } \\
\hline Yes & $17(46 \%)$ & $7(43.8 \%)$ & \multirow{2}{*}{0.8828} \\
\hline No & $20(54 \%$ & $9(56.3 \%)$ & \\
\hline \multicolumn{4}{|c|}{ Speech therapy adherence (post-operative) } \\
\hline Yes & $28(80 \%)$ & $13(86.7 \%)$ & \multirow{2}{*}{0.5739} \\
\hline No & $7(20 \%)$ & $2(13.3 \%)$ & \\
\hline \multicolumn{4}{|c|}{ Satisfaction } \\
\hline Yes & $38(100 \%)$ & $14(82.4 \%)$ & 0.0077 \\
\hline No & $0(0 \%)$ & $3(17.6 \%)$ & \\
\hline
\end{tabular}

$(P=0.0339, M c N e m a r$ 's test $)$. It showed an improvement of $50 \%$ within this group. Group B presented 2 (11.8\%) patients with vertigo before and 2 (11.8\%) 
patients after cochlear implantation $(P=1.00$, McNemar's test $)$.

There was a statistically significant improvement in group $A$ regarding social isolation after CI surgery $(P=0.0002$, chi-square test $)$. When groups were analyzed separately, there was a bigger difference in group $A(P=0.0009)$ than group $B(P=0.0588$, chi-square test; Table 5).

The same was observed about labor inclusion, comparing both groups-preand post-operatively. There was a general improvement $(P=0.0067$, chi-square test), with slightly better results for group $A$, after the surgery ( $P=0.0339 \mathrm{vs}$. 0.0833, chi-square test; Table 5).

User's satisfaction about the usage of the CI was verified by IOI-HA (International Outcome Inventory for Hearing Aids) [22]. There is an evident difference between groups, and it was more noticeable within the group with a shorter time of sound deprivation $(\mathrm{P}=0.0077)$.

\section{Discussion}

There is no statistical evidence related to the samples of this study regarding sex and age at the time of surgery, as published by Lazard et al. [7] and Blamey et al. [10].

Differences in auditory thresholds, before and after cochlear implantation, are significantly for both groups. It was already expected due to different technologies and applicability of the electronic device, as shown in the literature. The CI is the best option for severe to profound deafness, with great effectiveness for oral language and speech perception development, as demonstrated by many studies [23] [24] [25] [26].

Data available in the literature suggest that post-lingual deafened adults, with sound deprivation greater than 10 years, showed a worse performance on speech perception tests, after 1 and 2 years of the CI surgery. Despite of good results in both groups on sound detection, represented by auditory thresholds after cochlear implantation, group $A$ presented a better performance $(91.7 \pm 9.0)$ than group $B(78.8 \pm 17.1)$, similarly to Friedland et al., Portmann et al., Blamey et al., and Holden et al. [6] [8] [10] [27].

There was no significant difference among different brands of $\mathrm{CI}$, between groups $\mathrm{A}$ and $\mathrm{B}$, as well there was no difference regarding the etiology of hearing loss, what concurs with Lazard et al. who also did not observe differences regarding auditory outcomes among the different brands of CI, but found differences regarding the etiology of hearing loss [7].

Tinnitus was present, with statistical difference, within group $B$, which suggests that the greater the period of sound deprivation, the lower is the chance of tinnitus improvement after CI surgery. This may happen due to the habituation phenomenon. Despite this fact, there was improvement in tinnitus in both groups, reinforcing the effectiveness of CI surgery in improving tinnitus when is associated with hearing loss, as demonstrated by Kim et al., and Greenberg et al. [28] [29]. 
Vertigo was present in both groups, but group $A$ showed better outcomes regarding its resolution after cochlear implantation $(P=0.0339)$, differently of what happened in group $B$. The results of this study are accordingly to Chen et al. [30], and demonstrate that the shorter is the period of sound deprivation, the greater the possibility of improvement of vertigo after the CI surgery.

Social isolation and labor inclusion were statistically different between groups $\mathrm{A}$ and $\mathrm{B}$, what may indicate a negative correlation between these factors and the duration of sound deprivation. Furthermore, clinical experience has demonstrated the need to evaluate the individual's satisfaction of all performed interventions, and to associate them with clinical examination, to ensure an effective adaptation of the user and the device. Yet, self-evaluation questionnaires are important tools to support professionals during follow-up of individuals with CI devices as they provide information on the difficulties and facilities of the individual during their use [31]. For both groups, it could be documented the improvement in user's satisfaction and quality of life, and the reduced impact that hearing loss can cause.

The results of the present study confirm previous findings in the literature and support the relation between the greater is the period of sound deprivation, the worse is the performance of speech perception test after cochlear implantation.

Some factors must be considered as limitation for the data interpretation, such as the number of subjects enrolled that is related to the second limiting factor, the data acquisition from medical records and to be a retrospectively study. These factors were minimized by careful data acquisition and statistical analysis that demonstrates a homogeneous distribution between the both groups.

\section{Conclusions}

Adult sound deprivation greater than 10 years can lead to worse performance in speech perception test when compared to a shorter time of sound deprivation.

The longer time of sound deprivation also negatively influenced other factors such as tinnitus, vertigo and social isolation after the CI surgery.

Despite these findings, surgery in patients with sound deprivation greater than 10 years is not contraindicated.

\section{Conflict of Interest}

Nothing to declare.

\section{References}

[1] Bazon, A.C., et al. (2016) Auditory Speech Perception Tests in Relation to the Coding Strategy in Cochlear Implant. International Archives of Otorhinolaryngology, 20, 254-260. https://doi.org/10.1055/s-0035-1559595

[2] Gomez, M.V.S.G., et al. (2004) Medical and Audiological Selection Criteria and Evaluation for Cochlear Implants Candidates: HC-FMUSP protocol. International Archives of Otorhinolaryngology, 8, 295-303.

[3] Bento, R.F., et al. (2004) Auditory Results with the Multichannel Cochlear Implant 
in Patients Undergoing Surgery at the Hospital de las Clínicas of the Faculty of Medicine of the University of São Paulo. Revista Brasileira de Otorrinolaringologia, 9, 354-357.

[4] Hyppolito, M.Â. and Bento, R.F. (2012) Directions of the Bilateral Cochlear Implant Brazil. Brazilian Journal of Otorhinolaryngology, 78, 2-3. https://doi.org/10.1590/S1808-86942012000100001

[5] Zeitler, D.M., et al. (2009) Revision Cochlear Implantation. Current Opinion in Otolaryngology \& Head and Neck Surgery, 17, 334-338.

https://doi.org/10.1097/MOO.0b013e32832dd6ac

[6] Friedland, D.R., et al. (2003) Choice of Ear for Cochlear Implantation: The Effect of History and Residual Hearing on Predicted Postoperative Performance. Otology \& Neurotology, 24, 582-589. https://doi.org/10.1097/00129492-200307000-00009

[7] Lazard, D.S., et al. (2012) Pre-, Per- and Postoperative Factors Affecting Performance of Postlinguistically Deaf Adults Using Cochlear Implants: A New Conceptual Model over Time. PLoS One, 7, e48739.

https://doi.org/10.1371/journal.pone.0048739

[8] Portmann, D., et al. (2007) Bilateral Cochlear Implantation in a Patient with Long-Term Deafness. Revue de Laryngologie Otologie Rhinologie (Bord), 128, 65-68.

[9] Geier, L., et al. (1999) The Effect of Long-Term Deafness on Speech Recognition in Postlingually Deafened Adult CLARION Cochlear Implant Users. Annals of Otology, Rhinology \& Laryngology, 177, 80-83. https://doi.org/10.1177/00034894991080S416

[10] Blamey, P., et al. (1996) Factors Affecting Auditory Performance of Postlinguistically Deaf Adults Using Cochlear Implants. Audiology and Neurotology, 1, 293-306. https://doi.org/10.1159/000259212

[11] Plank, K., et al. (2016) Factors Predicting Postoperative Unilateral and Bilateral Speech Recognition in Adult Cochlear Implant Recipients with Acoustic Hearing. Ear Hear, 37, 153-163. https://doi.org/10.1097/AUD.0000000000000233

[12] Moon, I.S., et al. (2014) Is There a Deafness Duration Limit for Cochlear Implants in Post-Lingual Deaf Adults? Acta Oto-Laryngologica, 134, 173-180. https://doi.org/10.3109/00016489.2013.850175

[13] Baskill, J.L. and Coles, R.R.A. (1999) Relationship between Tinnitus Loudness and Severity. In: Hazell, J., Ed., Sixth International Tinnitus Seminar, Cambridge, UK, 424-428.

[14] Ferrari, G.M.S., et al. (2007) The Efficacy of Open Molds in Controlling Tinnitus. Revista Brasileira de Otorrinolaringologia, 73, 370-377. https://doi.org/10.1590/S0034-72992007000300012

[15] Sanchez, T.G., et al. (2005) Tinnitus in Normally Hearing Patients: Clinical Aspects and Repercussions. Revista Brasileira de Otorrinolaringologia, 71, 427-431. https://doi.org/10.1590/S0034-72992005000400005

[16] Silva, R., et al. (2007) Hearing Aid: Does It Reduce the Sensation of Tinnitus? Revista CEFAC, 9, 263-268. https://doi.org/10.1590/S1516-18462007000200016

[17] Pinto, P.C.L., et al. (2010) The Impact of Gender, Age and Hearing Loss on Tinnitus Severity. Brazilian Journal of Otorhinolaryngology, 76, 18-24. https://doi.org/10.1590/S1808-86942010000100004

[18] Van der Berge, M.J.C., et al. (2017) Effect of Direct Stimulation of the Cochleovestibular Nerve on Tinnitus: A Long-Term Follow-Up Study. World Neurosurgery, 98, 571-577. 
[19] Tugumia, D., et al. (2016) Auditory Training Program in Subjects with Tinnitus. CoDAS, 28, 27-33. https://doi.org/10.1590/2317-1782/20162015113

[20] Mondelli, M.F.C.G., et al. (2016) Correlation between Speech Perception and Tinnitus before and after the Use of Hearing Aids. Audiology-Communication Research, 21, e1649.

[21] Moura, L.O.S., et al. (2004) The Effectiveness of Hearing Aid Fitting in Reducing or Eliminating the Tinnitus. Revista Brasileira de Otorrinolaringologia, 70, 624-631. https://doi.org/10.1590/S0034-72992004000500008

[22] Cox, R.M. and Alexander, G.C. (2002) The International Outcome Inventory for Hearing Aids (IOI-HA): Psychometric Properties of the English Version. International Journal of Audiology, 41, 30-35. https://doi.org/10.3109/14992020209101309

[23] Miziara, I.D., et al. (2012) Bioethics and Medical/Legal Considerations on Cochlear Implants in Children. Brazilian Journal of Otorhinolaryngology, 78, 70-79. https://doi.org/10.1590/S1808-86942012000300013

[24] Moret, A.L.M., et al. (2007) Cochlear Implant: Hearing and Language in Pre-Lingual Deaf Children. Pró-Fono Revista de Atualização Científica, 19, 295-304. https://doi.org/10.1590/S0104-56872007000300008

[25] Angelo, T.C.S., et al. (2010) Speech Perception in Pre-Lingual Deaf Users of Cochlear Implant. Pró-Fono Revista de Atualização Científica, 22, 275-280. https://doi.org/10.1590/S0104-56872010000300020

[26] Queiroz, C.A.U.F., et al. (2010) Longitudinal Study of Verbal Comprehension of Children with Cochlear Implant. Revista CEFAC, 12, 210-215. https://doi.org/10.1590/S1516-18462010000200006

[27] Holden, L.K., et al. (2013) Factors Affecting Open-Set Word Recognition in Adults with Cochlear Implants. Ear Hear, 34, 342-360. https://doi.org/10.1097/AUD.0b013e3182741aa7

[28] Kim, D.K., et al. (2016) Prospective, Multicenter Study on Tinnitus Changes after Cochlear Implantation. Audiology and Neurotology, 21, 165-171. https://doi.org/10.1159/000445164

[29] Greenberg, D., et al. (2015) Developing an Assessment Approach for Perceptual Changes to Tinnitus Sound Characteristics for Adult Cochlear Implant Recipients. International Journal of Audiology, 55, 392-404. https://doi.org/10.3109/14992027.2016.1172391

[30] Chen, X., et al. (2016) Influence of Cochlear Implantation on Vestibular Function. Acta Oto-Laryngologica, 136, 655-659. https://doi.org/10.3109/00016489.2016.1154186

[31] Rosa, M.R.D., et al. (2006) Hearing Aid Program and Self-Assessment Questionnaires: Importants Instruments for Effective Hearing Adaptation. Arq Intern Otorrinolaringol, 10, 220-227.
Abbreviations
$\mathrm{CI}=$ cochlear implant
$\mathrm{HA}=$ hearing aid
SNHL $=$ sensorineural hearing loss 